\title{
One approach to the problems of impact of fine shells of the S.P. Timoshenko type on elastic half-space
}

\author{
Vladislav Bogdanov \\ Progressive Research Solutions Pty. Ltd. \\ 28/2 Buller Rd, Artarmon, Sydney, Australia 2064 \\ vladislav_bogdanov@hotmail.com, orcid.org/0000-0002-3424-1801 \\ Received 06.02.2021, accepted after revision 06.07.2021 \\ https://doi.org/10.32347/uwt2021.11.1101
}

\begin{abstract}
Shell elements are used in many thinwalled structures. Therefore, to study the dynamics of propagation of wave processes in the fine shells of S.P. Timoshenko type is an important aspect as well as it is important to investigate a wave processes of the impact, shock in elastic foundation in which a striker is penetrating. Refined model of S.P. Timoshenko makes it possible to consider the shear and the inertia rotation of the transverse section of the shell. Disturbances spread in the shells of S.P. Timoshenko type with finite speed. The method of the outcoming dynamics problems to solve an infinite system of integral equations Volterra of the second kind and the convergence of this solution are well studied. Such approach has been successfully used for cases of the investigation of problems of the impact a hard bodies and an elastic fine shells of the KirchhoffLove type on elastic a half-space and a layer. In this paper an attempt is made to solve the plane and the axisymmetric problems of the impact of an elastic fine cylindric and spheric shells of the S.P. Timoshenko type on an elastic half-space using the method of the outcoming dynamics problems to solve an infinite system of integral equations Volterra of the second kind. The discretization using the Gregory methods for numerical integration and Adams for solving the Cauchy problem of the reduced infinite system of Volterra equations of the second kind results in a poorly defined system of linear algebraic equations: as the size of reduction increases the determinant of such a system to aim at infinity. This technique does not allow to solve plane and axisymmetric problems of dynamics for fine shells of the S.P. Timoshenko type and elastic bodies. It is shown that this approach is not acceptable for investigated in this paper the plane and the axisymmetric problems.
\end{abstract}

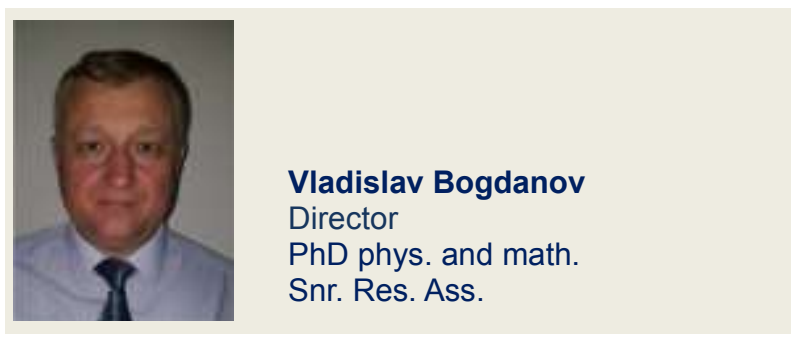

This shows the limitations of this approach and leads to the feasibility of developing other mathematical approaches and models. It should be noted that to calibrate the computational process of deformation in the elastoplastic formulation at the elastic stage, it is convenient and expedient to use the technique of the outcoming dynamics problems to solve an infinite system of integral equations Volterra of the second kind.

Keywords: impact, elastic, elastic-plastic, halfspace, axisymmetric problem, fine, spherical shell, S.P. Timoshenko.

\section{INTRODUCTION}

The approach [2-6] for solving problems of dynamics, developed in [7 -9, 11], makes it possible to determine the stress-strain state of elastic half-space and a layer during penetration of absolutely rigid bodies $[2,3,8$, $9,11]$ and the stress-strain state of elastic Kirchhoff-Love type fine shells and elastic half-spaces and layers at their collision [4-7]. This led to the feasibility of developing other mathematical approaches and models. In [10, 12 - 15], a new approach to solving the problems of impact and nonstationary interaction in the elastoplastic mathematical 
formulation [16 - 20] was developed. In nonstationary problems, the action of the striker is replaced by a distributed load in the contact area, which changes according to a linear law [21 - 23]. The contact area remains constant. The developed elastoplastic formulation makes it possible to solve impact problems when the dynamic change in the boundary of the contact area is considered and based on this the movement of the striker as a solid body with a change in the penetration speed is taken into account. Also, such an elastoplastic formulation makes it possible to consider the hardening of the material in the process of nonstationary and impact interaction.

The solution of problems for elastic shells [24 - 27], elastic half-space [28 - 30], elastic layer [31], elastic rod [32, 33] were developed using method of the influence functions [34]. In [24] the process of non-stationary interaction of an elastic cylindrical shell with an elastic half-space at the so-called "supersonic" stage of interaction is studied. It is characterized by an excess of the expansion rate areas of contact interaction speed of propagation tension-compression waves in elastic half-space. The solution was developed using influence functions corresponding concentrated force or kinematic actions for an elastic isotropic half-space which were found and investigated in [34].

In this paper, we investigate the approach [4 - 7] for solving the axisymmetric problem of the impact of a spherical fine shell of the S.P. Timoshenko type on an elastic half-space.

It is shown that the approach [2-5], after the reduction of the infinite system of Volterra integral equations of the second kind $[6-8$, 11] and discretization using the Gregory methods for numerical integration and Adams for solving the Cauchy problem, a poorly defined system of linear algebraic equations is obtained for which the determinant of the matrix of coefficients increases indefinitely with increasing size of reduction.

\section{PROBLEM FORMULATION}

A thin elastic cylindrical shell comes into collision with the elastic half-space $z \geq 0$ with its lateral surface along the generatrix of the cylinder at the moment of time $t=0$. We associate with the shell, as can be seen in Figure 1, a movable cylindrical coordinate system $r \theta z^{\prime}: \theta$ - the polar angle, which is plotted from the positive direction of the $o z$ axis, the $o y$ axis coincides with the cylinder axis. Let us denote by $u_{0}(t, \theta), w_{0}(t, \theta)$, $p(t, \theta), q(t, \theta)$ the tangential and normal displacements of the points of the middle surface of the shell and the radial and tangential components of the distributed external load, which acts on the shell. We associate a fixed Cartesian coordinate system $x y z$ with the half-space, so that the $O z$ axis is directed deep into the medium, the $O x$ axis is directed along the surface of the half-space, and the $O y$ axis is parallel to the generatrix of the cylinder. The shell thickness $h$ is much less than the radius $R$ of the middle surface of the shell $(h / R \leq 0,05)$.

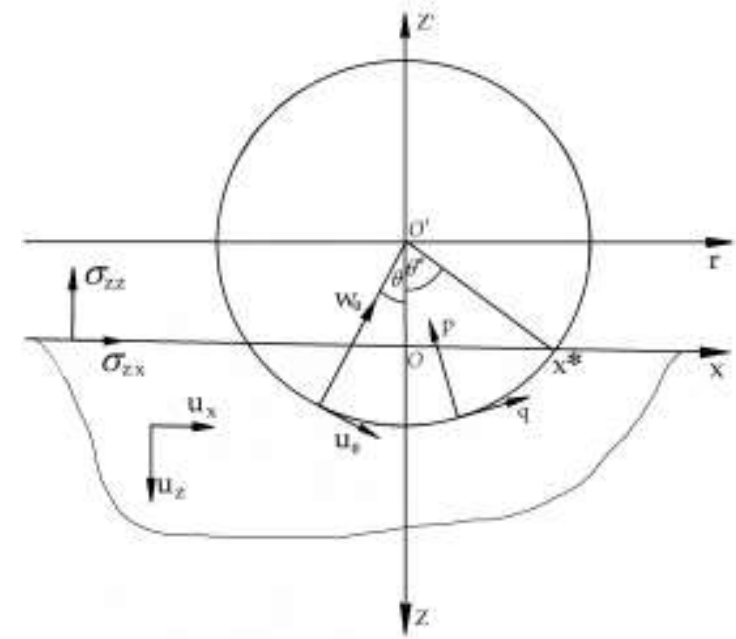

Fig. 1. Scheme of the system cylindrical shell - half space

In case of axisymmetric problem, a thin elastic spherical shell, moving perpendicular to the surface of the elastic half-space $z \geq 0$, reaches this surface at time $t=0$. We associate with the shell, as shown in Fig. 2, a movable spherical coordinate system $r^{\prime} \varphi^{\prime} \theta$, where $\varphi^{\prime}-$ is the longitude of the radius vector $r, \theta-$ is the polar angle.

With the half-space we associate a fixed cylindrical coordinate system $r \varphi z$, the $O z$ axis is directed deep into the medium, $\varphi-$ is the 
polar angle. Angle $\theta$ is plotted from the positive direction of the $\mathrm{Oz}$ axis.

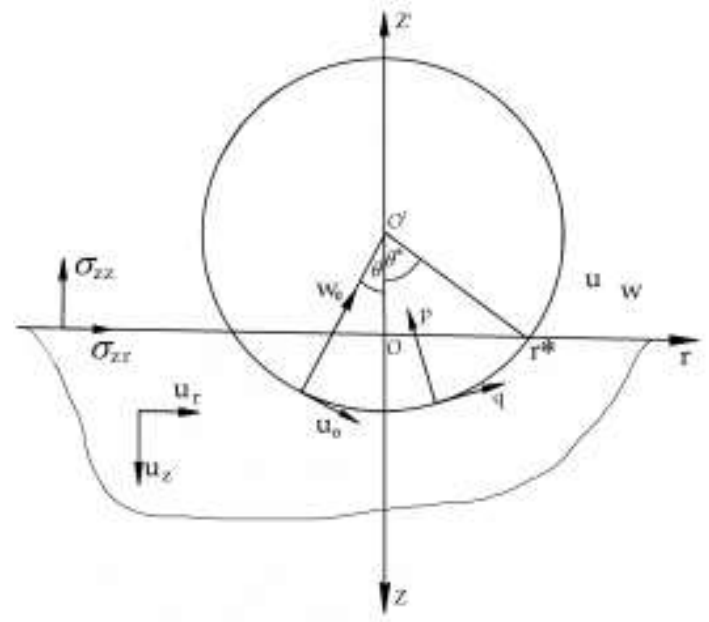

Fig. 2. Scheme of the system spherical shell - half space

The cylindric or spheric shell penetrates into the elastic medium at a speed $v_{T}(t),(0 \leq t \leq T)$, the initial penetration rate is $V_{0}=v_{T}(0), T$ - the time during which the shell interacts with the half-space. The shell thickness $h$ is much less than the radius $R$ of the middle surface of the shell $(h / R \leq 0,05)$.

Let us denote by $u_{0}(t, \theta), w_{0}(t, \theta), p(t, \theta)$, $q(t, \theta)$ the tangential and normal displacements of the points of the middle surface of the shell and the radial and tangential components of the distributed external load, which acts on the shell. With the half-space we associate a fixed cylindrical coordinate system $r \varphi z$, the $O z$ axis is directed deep into the medium, $\varphi-$ is the polar angle. Angle $\theta$ is plotted from the positive direction of the $\mathrm{Oz}$ axis. The physical properties of the half-space material are characterized by elastic constants: volumetric expansion module $K$, shear modulus $\mu$ and density $\rho$. An elastic medium with constants $K, \mu, \rho$ will be associated with a hypothetical acoustic medium with the same constants $K, \rho$, wherein $\mu=0$. Under $C_{p}, C_{S}, C_{0}$ we mean the speed of longitudinal and transverse waves in an elastic half-space and the speed of sound in the considered hypothetical acoustic medium.
Let's introduce dimensionless variables:

$$
\begin{aligned}
& t^{\prime}=\frac{C_{0} t}{R},\left\|\begin{array}{l}
x^{\prime} \| \\
r^{\prime}
\end{array}\right\|=\frac{1}{R}\|r\|, \quad z^{\prime}=\frac{z}{R}, \quad u_{i}^{\prime}=\frac{u_{i}}{R}, \\
& u_{0}^{\prime}=\frac{u_{0}}{R}, \quad w_{0}^{\prime}=\frac{w_{0}}{R}, \quad \sigma_{i j}^{\prime}=\frac{\sigma_{i j}}{K}, \quad v_{T}^{\prime}=\frac{v_{T}}{C_{0}}, \\
& w_{T}^{\prime}=\frac{w_{T}}{R}, \quad p^{\prime}=\frac{p}{K R}\left\|\begin{array}{c}
1 \\
1 / R
\end{array}\right\|, \quad q^{\prime}=\frac{q}{K R}\left\|\begin{array}{c}
1 \\
1 / R
\end{array}\right\|, \\
& M^{\prime}=\frac{M}{\rho R^{2}}\left\|\begin{array}{c}
1 \\
1 / R
\end{array}\right\| \cdot\left(i, j=\left\|\begin{array}{l}
x \\
r
\end{array}\right\|,\left\|\begin{array}{l}
y \\
\varphi
\end{array}\right\|, z\right) \\
& \beta^{2}=\frac{C_{S}^{2}}{C_{0}^{2}}=\frac{\mu}{K}, \alpha^{2}=\frac{C_{p}^{2}}{C_{0}^{2}}=\left(1+\frac{4 \mu}{3 K}\right), \\
& C_{0}^{2}=\frac{K}{\rho}, b^{2}=\frac{\beta^{2}}{\alpha^{2}}=\frac{3 \mu}{3 K+4 \mu} .
\end{aligned}
$$

here $\mathbf{u}=\left(u_{\| x \mid}, u_{r \mid},\left|\begin{array}{l}\mid y \\ \varphi\end{array}\right|, u_{z}\right)-$ is the vector of movement of points of the environment; $\sigma_{z z}, \sigma_{\|x z\|}-$ nonzero components of the stress tensor of the medium; $M$ - is the shell running mass; $v_{T}(t), w_{T}(t)$ - speed and movement of the shell as a solid. In what follows, we will use only dimensionless quantities, so we omit the dash. The elastic half-space and the spheric shell are in a state of axisymmetric deformation.

Differential equations (of the S.P. Timoshenko type) describing the dynamics of cylindrical (2) and spherical (3) shells and considering the shear and inertia of rotation of the transverse section, due to (1), take the following form [35, pp. 297, 307]:

$\gamma_{0}^{2} \frac{\partial^{2} u_{0}}{\partial t^{2}}=\frac{\partial^{2} u_{0}}{\partial \theta^{2}}+\left(1+a_{4}\right) \frac{\partial w_{0}}{\partial \theta}+a_{4} \Phi-$

$-a_{4} u_{0}+\beta_{3} q$,

$\eta_{0}^{2} \frac{\partial^{2} w_{0}}{\partial t^{2}}=\frac{\partial^{2} w_{0}}{\partial \theta^{2}}+\frac{\partial \Phi}{\partial \theta}-\left(1+a_{3}\right) \frac{\partial u_{0}}{\partial \theta}-$

$-a_{3} w_{0}+\beta_{4} p$,

$\gamma_{0}^{2} \frac{\partial^{2} \Phi}{\partial t^{2}}=\frac{\partial^{2} \Phi}{\partial \theta^{2}}-a_{2} \frac{\partial w_{0}}{\partial \theta}-a_{2} \Phi+a_{2} u_{0}$, 
$\frac{1}{1-v_{0}^{2}} \frac{\partial^{2} u_{0}}{\partial \theta^{2}}+\frac{\operatorname{ctg} \theta}{1-v_{0}^{2}} \frac{\partial u_{0}}{\partial \theta}+\frac{2\left(1+v_{0}\right) k_{s}+1-v_{0}}{2\left(1-v_{0}^{2}\right) k_{s}} \frac{\partial w_{0}}{\partial \theta}-$ $-\frac{v_{0}+\left(1-v_{0}\right) \cos ^{2} \theta}{\left(1-v_{0}^{2}\right) \sin ^{2} \theta} u_{0}+\frac{\Phi}{2\left(1+v_{0}\right) k_{s}}=\gamma_{0}^{2} \frac{\partial^{2} u_{0}}{\partial t^{2}}-q$, $\frac{1}{2\left(1+v_{0}\right) k_{s}} \frac{\partial^{2} w_{0}}{\partial \theta^{2}}-\frac{1}{1-v_{0}} \frac{\partial u_{0}}{\partial \theta}+\frac{\operatorname{ctg} \theta}{2\left(1+v_{0}\right) k_{s}} \frac{\partial w_{0}}{\partial \theta}+$ $+\frac{1}{2\left(1+v_{0}\right) k_{s}} \frac{\partial \Phi}{\partial \theta}-\frac{\operatorname{ctg} \theta}{1-v_{0}} u_{0}-\frac{2}{1-v_{0}} w_{0}+$

$+\frac{\operatorname{ctg} \theta}{2\left(1+v_{0}\right) k_{s}} \Phi=\gamma_{0}^{2} \frac{\partial^{2} w_{0}}{\partial t^{2}}-p$,

$\frac{\partial^{2} \Phi}{\partial \theta^{2}}+\operatorname{ctg} \theta \frac{\partial \Phi}{\partial \theta}-\frac{E_{0} h R^{2}}{2\left(1+v_{0}\right) k_{s} D} \frac{\partial w_{0}}{\partial \theta}-$

$-\frac{\left(1-v_{0}\right) k_{s} D\left(2 v_{0}+\left(1-v_{0}\right) \sin 2 \theta\right)+E_{0} h R^{2} \sin ^{2} \theta}{2\left(1+v_{0}\right) k_{s} D \sin ^{2} \theta} \Phi=$ $=\eta_{0}^{2} \frac{\partial^{2} \Phi}{\partial t^{2}}$

where

$$
\begin{aligned}
& \gamma_{0}^{2}=\left\|\begin{array}{c}
C_{0}^{2} / C_{02}^{2} \\
\rho_{0} k_{1} C_{0}^{2} / E_{0}
\end{array}\right\|, \eta_{0}^{2}=\left\|\begin{array}{c}
C_{0}^{2} / C_{01}^{2} \\
\rho_{0} h^{3} C_{0}^{2} k_{r} / 12 D
\end{array}\right\|, \\
& C_{01}^{2}=\frac{E_{0}}{\left(1-v_{0}^{2}\right) \rho_{0}}, C_{02}^{2}=\frac{b_{1}^{2} E_{0}}{2\left(1+v_{0}\right)}, \\
& a_{2}=\frac{6\left(1-v_{0}\right) b_{1}^{2} R^{2}}{h^{2}}, a_{3}=\frac{2}{\left(1-v_{0}\right) b_{1}^{2}}, \\
& \beta_{3}=\frac{\left(1-v_{0}^{2}\right) K^{2} R}{E_{0}^{2} h}, \beta_{4}=\frac{2\left(1+v_{0}\right) K^{2} R}{b_{1}^{2} E_{0}^{2} h} \\
& b_{1}^{2}=\frac{5}{6}, a_{4}=\frac{1}{a_{3}}, \quad k_{1}=1+\frac{h^{2}}{12 R^{2}}, \\
& k_{r}=1+\frac{3 h^{2}}{20 R^{2}}, \quad D=\frac{E_{0} h^{3}}{12\left(1-v_{0}^{2}\right)}, k_{s}=\frac{5}{6}
\end{aligned}
$$

here $\Phi$ - angle of rotation of the normal section to the middle surface, $b_{1}^{2}$ - coefficient that considers the distribution of tangential forces in the transverse section of the cylindrical shell, $k_{S}$ - shear ratio of the spherical shell, $D$ - cylindrical stiffness, $v_{0}, E_{0}, \rho_{0}-$ Poisson's ratio, Young's modulus and density of the shell material, $p$ и $q-$ respectively, the radial and tangential components of the distributed load acting on the shell, $R-$ is the shell radius.

The motion of an elastic medium is described by scalar potential $\varphi$ and non-zero component of vector potential $\psi$, which satisfy the wave equations [2-5]:

$$
\begin{gathered}
\Delta \varphi=\frac{\partial^{2} \varphi}{\alpha^{2} \partial t^{2}}, \Delta \psi=\frac{\partial^{2} \psi}{\beta^{2} \partial t^{2}}, \\
\Delta \equiv\left\|\begin{array}{c}
\frac{\partial^{2}}{\partial x^{2}}+\frac{\partial^{2}}{\partial y^{2}} \\
\frac{\partial}{2}_{\partial r^{2}}+\frac{\partial}{r \partial r}+\frac{\partial^{2}}{\partial z^{2}} \|
\end{array}\right\|
\end{gathered}
$$

Physical quantities are expressed in terms of wave potentials as follows [6-9]:

$$
\begin{gathered}
u_{x}=\frac{\partial \varphi}{\partial x}+\frac{\partial \psi}{\partial z}, u_{z}=\frac{\partial \varphi}{\partial z}-\frac{\partial \psi}{\partial x}, u_{y}=0, \\
\sigma_{z z}=\left(1-2 b^{2}\right) \frac{\partial^{2} \varphi}{\partial t^{2}}+2 \beta^{2} \times \\
\times \|\left(\frac{\partial^{2} \varphi}{\partial z^{2}}+\frac{\partial^{2} \psi}{\partial x \partial z}\right) \\
\times \\
\left.\sigma_{x z}=2 \beta^{2} \frac{\partial^{2} \varphi}{\partial x \partial z}+\frac{\partial^{2} \psi}{\partial z^{2}}+\frac{\partial \psi}{r \partial z}\right) \|, \\
\sigma_{x y}=\sigma_{y z}=0, \sigma_{x x}=\Theta-\sigma_{z z}, \\
u_{r}=\frac{\partial \varphi}{\partial r}-\frac{\partial \psi}{\partial z}, u_{z}=\frac{\partial \varphi}{\partial z}+\frac{\partial \psi}{\partial r}+\frac{\psi}{r}, \\
u_{\varphi}=0, \sigma_{r \varphi}=\sigma_{\varphi z}=0, \\
\sigma_{r z}=2 \beta^{2} \frac{\partial^{2} \varphi}{\partial r \partial z}+\frac{\partial^{2} \psi}{\partial t^{2}}-2 \beta^{2} \frac{\partial^{2} \psi}{\partial z^{2}}, \\
\sigma_{r r}=\left(1-2 b^{2}\right) \frac{\partial^{2} \varphi}{\partial t^{2}}+2 \beta^{2}\left(\frac{\partial^{2} \varphi}{\partial r^{2}}-\frac{\partial^{2} \psi}{\partial r \partial z}\right), \\
\sigma_{z z}+\sigma_{x x}=2\left(1-b^{2}\right) \frac{\partial^{2} \varphi}{\partial t^{2}} .
\end{gathered}
$$


If the shear modulus $\mu$ is set equal to zero $\mu=0$, then the equations of motion of the elastic medium will be the equations of acoustics.

Let us consider the initial stage of the process of impact of elastic shells on the surface of an elastic half-space [4 -7], when no plastic deformations occur and the depth of the shell penetration into the medium is small.

The problem of interaction of elastic shells with an elastic half-space is solved in a linear formulation, therefore, we linearize the boundary conditions $[2,3,8,9,11]$ : we transfer the boundary conditions from the perturbed surface to the undisturbed surface of the bodies that are deformed. We assume that there is no friction between the elastic halfspace and the penetrating body, or the slippage condition is valid.

As can be seen from Fig. 1, the projections of the functions $u_{0}, w_{0}, p$ and $q$ on the $\left\|\begin{array}{l}o^{\prime} r \\ o r\end{array}\right\|$ and $o z$ axes will be equal:

$$
\begin{aligned}
& \operatorname{pr}_{Z^{\prime}} w_{0}(t, \theta)=w_{0}(t, \theta) \cos \theta, \\
& \operatorname{pr}_{Z^{\prime}} u_{0}(t, \theta)=u_{0}(t, \theta) \sin \theta, \\
& \operatorname{pr}_{Z^{\prime}} p(t, \theta)=p(t, \theta) \cos \theta, \\
& \operatorname{pr}_{Z^{\prime}} q(t, \theta)=q(t, \theta) \sin \theta, \\
& \operatorname{pr}_{r} w_{0}(t, \theta)=-w_{0}(t, \theta) \sin \theta, \\
& \operatorname{pr}_{r} u_{0}(t, \theta)=u_{0}(t, \theta) \cos \theta, \\
& \operatorname{pr}_{r} p(t, \theta)=-p(t, \theta) \sin \theta, \\
& \operatorname{pr}_{r} q(t, \theta)=q(t, \theta) \cos \theta .
\end{aligned}
$$

Then, in the $\left\|\begin{array}{l}z o x \\ z o r\end{array}\right\|$ coordinate system, the displacements $u_{z}, u_{\|x\|}$ and stresses $\sigma_{z z}$ and $\sigma_{\| z x}$ at the surface points of the contact area will be written as:

$$
\begin{aligned}
& u_{z}\left(t,\left\|\begin{array}{l}
x \\
r
\end{array}\right\|, 0\right)=w_{T}(t)-f\left(\left\|\begin{array}{l}
x \| \\
r
\end{array}\right\|\right)-w_{0}(t, \theta) \cos \theta- \\
& -u_{0}(t, \theta) \sin \theta,
\end{aligned}
$$

$$
\begin{aligned}
& u_{x}(t, x, 0)=-w_{0}(t, \theta) \sin \theta+u_{0}(t, \theta) \cos \theta, \\
& u_{r}(t, r, 0)=-w_{0}(t, \theta) \sin \theta+u_{0}(t, \theta) \cos \theta, \\
& \sigma_{z z}\left(t,\|x\|_{r}, 0\right)=-p(t, \theta) \cos \theta-q(t, \theta) \sin \theta, \\
& \sigma_{r z}(t, r, 0)=-p(t, \theta) \sin \theta+q(t, \theta) \cos \theta, \\
& p(t, \theta)=-\sigma_{z z}(t,\|x\|, 0) \cos \theta-\sigma_{\| x z}(t,\|x\|, 0) \sin \theta,|\theta|<\theta^{*}, \\
& q(t, \theta)=-\sigma_{z z}\left(t,\|x\|_{r z} \|, 0\right) \sin \theta+\sigma_{\| x z}(t,\|x\|, \|, 0) \cos \theta,|\theta|<\theta^{*},
\end{aligned}
$$

where $w_{T}(t)$ - displacement of the shell as a rigid body, the function $f()$ describes the shell profile, $2 \theta^{*}$ as can be seen from Figures 1 and 2 , the size of the shell sector in contact with the half-space. In the case of the cylindrical and the spherical shells:

$$
f(\|x\|)=1-\sqrt{1-\left\|\begin{array}{l}
x^{2} \\
r^{2}
\end{array}\right\|}
$$

The kinematic condition that determines the half-size of the contact area $\left\|\begin{array}{l}x^{*} \\ r^{*}\end{array}\right\|(t)$ is written as follows:

$$
\begin{aligned}
& w_{T}(t)-f(\|x\|)-u_{z}(t,\|x\|, 0)-w_{0}(t, \theta) \cos \theta- \\
& -u_{0}(t, \theta) \sin \theta= \begin{cases}0, & \text { if }\|x\| \leq \| \begin{array}{l}
x^{*} \| \\
r^{*} \|
\end{array}(t), \\
\varepsilon<0, & \text { if }\|x\|>\left\|x^{*}\right\|(t),\end{cases}
\end{aligned}
$$

We assume that the contact area is simply connected region, and this statement is equivalent to the fact that the stresses normal to the contact area are compressive:

$$
\left.\sigma_{z z}\right|_{z=0}<0,\left\|\begin{array}{l}
\mid x \\
r
\end{array}\right\|<\left\|\begin{array}{l}
x^{*} \| \\
r^{*}
\end{array}\right\|(t) .
$$

Based on (5), the boundary conditions in the absence of friction in the contact zone can be formulated as follows: 


$$
\begin{aligned}
& \left.\frac{\partial u_{z}}{\partial t}\right|_{z=0} \equiv V\left(t,\left\|\begin{array}{l}
x \| \\
r \|
\end{array}\right\|=v_{T}(t)-\frac{\partial w_{0}(t, \theta)}{\partial t} \cos \theta-\right. \\
& -\frac{\partial u_{0}(t, \theta)}{\partial t} \sin \theta,\left\|\begin{array}{c}
x \| \\
r
\end{array}\right\|<\left\|\begin{array}{c}
x^{*} \\
r^{*}
\end{array}\right\|(t), \\
& \left.\sigma_{z z}\right|_{z=0}=0,\left\|\begin{array}{l}
\mid x \| \\
r
\end{array}\right\|>\left\|\begin{array}{l}
x^{*} \\
r^{*}
\end{array}\right\|(t), \quad \sigma_{\| z x}\left\|\left._{z r}\right|_{z=0}=0, \quad\right\| \begin{array}{c}
|x|<\infty \\
r>0
\end{array} \| .
\end{aligned}
$$

The initial conditions for potentials and are zero:

$$
\left.\varphi\right|_{t=0}=\left.\frac{\partial \varphi}{\partial t}\right|_{t=0}=0,\left.\quad \psi\right|_{t=0}=\left.\frac{\partial \psi}{\partial t}\right|_{t=0}=0 .
$$

For the problem of impact of an elastic shell on an elastic half-space, the velocity and displacement of the impacting body are found from the equation of motion by integrating it.

The equation of motion of a shell of mass $M$ for the problem of impact with an initial velocity $V_{0}$ has the form:

$$
\begin{aligned}
& M \frac{d^{2} w_{T}(t)}{d t^{2}}=-P(t), \\
& \left.v_{T}(t)\right|_{t=0}=V_{0},\left.\quad w_{T}(t)\right|_{t=0}=0, \\
& P(t)=-2\|\|_{\pi r}\left\|\int_{0}^{\left\|x^{*}\right\|} \sigma_{z z}^{r^{*} \|}(t,\|x\|, 0) d\right\| x \| .
\end{aligned}
$$

The condition for the absence of disturbances ahead of the front of longitudinal waves and the condition for damping of disturbances at infinity are valid.

$$
\begin{aligned}
& \left.\varphi\right|_{\rho_{1}>\alpha t+C_{\alpha}}=0,\left.\quad \psi\right|_{\rho_{1}>a t+C_{\alpha}}=0, \\
& \left.\varphi\right|_{\rho_{1} \rightarrow \infty} \rightarrow 0,\left.\quad \psi\right|_{\rho_{1} \rightarrow \infty} \rightarrow 0,
\end{aligned}
$$

where $\rho_{1}=\left\|\begin{array}{l}x^{2} \\ r^{2}\end{array}\right\|+z^{2}, \quad C_{\alpha}=$ const.

\section{SOLUTION ALGORITHM}

Since the impact process is short-term, the perturbation region at each moment of time $t$ is finite. Restricting ourselves to a finite interval of interaction time $(0 \leq t \leq T)$, it is possible to select a region of a half-space, which by the time moment $T$ covers the entire zone of disturbances. From this point of view, for times $(0 \leq t \leq T)$, the elastic half-space can be replaced by an elastic half-strip $(|x| \leq l ; z \geq 0)$ in case of plane problem and half-cylinder $(r \leq l ; z \geq 0)$ in case of axisymmetric problem, the boundaries of which do not reach the perturbations by the time $T$.

$$
l=\alpha T+\left\|\begin{array}{l}
x^{*} \| \\
r^{*}
\end{array}\right\|(T)
$$

Thus, for times $(0 \leq t \leq T)$, the considered problem is reduced to a nonstationary problem for a half-strip and a half-cylinder with mixed boundary conditions at its end. To represent the displacement vector as:

$$
u=\operatorname{grad} \varphi+\operatorname{rot} \psi, \operatorname{div} \psi=0,
$$

on the lateral surface of the half-strip and the half-cylinder, we select, for example, the conditions for sliding termination:

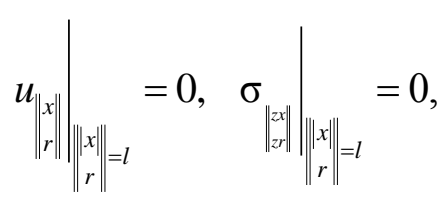

or

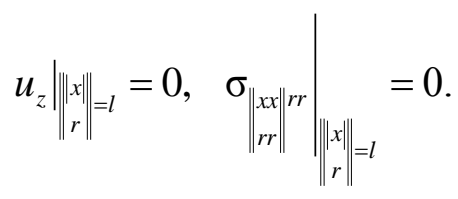

Consider the initial - boundary value problem (2) or (3), (4), (9) - (12). Let us represent the normal $w_{0}(t, \theta)$ and tangential $u_{0}(t, \theta)$ displacements of the points of the middle surface of the shell and the radial $p(t, \theta)$ and tangential $q(t, \theta)$ components of the distributed external load acting on the shell in the form of trigonometric Fourier series in case of plane problem and in Legendre polynomials and their derivatives in case of axisymmetric problem. 


$$
\begin{aligned}
& w_{0}(t, \theta)=\sum_{n=0}^{\infty} w_{0 n}(t)\left\|\begin{array}{c}
\cos (n \theta) \\
P_{n}(\cos \theta)
\end{array}\right\|, \\
& u_{0}(t, \theta)=\sum_{n=1}^{\infty} u_{0 n}(t)\left\|\begin{array}{c}
\sin (n \theta) \\
P_{n}^{1}(\cos \theta)
\end{array}\right\|, \\
& p(t, \theta)=\sum_{n=0}^{\infty} p_{n}(t)\left\|\begin{array}{c}
\cos (n \theta) \\
P_{n}(\cos \theta)
\end{array}\right\|, \\
& q(t, \theta)=\sum_{n=1}^{\infty} q_{n}(t)\left\|\begin{array}{c}
\sin (n \theta) \\
P_{n}^{1}(\cos \theta)
\end{array}\right\|, \\
& \Phi(t, \theta)=\sum_{n=1}^{\infty} \Phi_{n}(t)\left\|\begin{array}{c}
\sin (n \theta) \\
P_{n}^{1}(\cos \theta)
\end{array}\right\|
\end{aligned}
$$

In the space of Laplace transformants with parameter $s$, the transformants of functions $\Phi, w_{0}, u_{0}, p, q$ will, due to (18) - (22), have the form:

$$
\begin{gathered}
w_{0}^{L}(s, \theta)=\sum_{n=0}^{\infty} w_{0 n}^{L}(s)\left\|\begin{array}{c}
\cos (n \theta) \\
P_{n}(\cos \theta)
\end{array}\right\|, \\
u_{0}^{L}(s, \theta)=\sum_{n=1}^{\infty} u_{0 n}^{L}(s)\left\|\begin{array}{c}
\sin (n \theta) \\
P_{n}^{1}(\cos \theta)
\end{array}\right\|, \\
p^{L}(s, \theta)=\sum_{n=0}^{\infty} p_{n}^{L}(s)\left\|\begin{array}{c}
\cos (n \theta) \\
P_{n}(\cos \theta)
\end{array}\right\|, \\
q^{L}(s, \theta)=\sum_{n=1}^{\infty} q_{n}^{L}(s)\left\|\begin{array}{c}
\sin (n \theta) \\
P_{n}^{1}(\cos \theta)
\end{array}\right\|, \\
\Phi^{L}(s, \theta)=\sum_{n=1}^{\infty} \Phi_{n}^{L}(s)\left\|\begin{array}{c}
\sin (n \theta) \\
P_{n}^{1}(\cos \theta)
\end{array}\right\| .
\end{gathered}
$$

We apply to the system of equations (2) and (3) the Laplace transform in the variable $t$ with the parameter $s$ and substitute their equalities (23) - (27). Equating the coefficients at the same $\cos (n \theta)$ and $\sin (n \theta)$ in case of plane problem and $P_{n}(\cos \theta)$ and $P_{n}^{1}(\cos \theta)$ in case of axisymmetric problem we obtain the relations connecting the components of the expansion into series of functions $\Phi^{L}, w_{0}^{L}, u_{0}^{L}, p^{L}$ and $q^{L}$.

$$
w_{0,0}^{L}(s)=\left\|\begin{array}{c}
\frac{\beta_{4} p_{0}^{L}(s)}{\eta_{0}^{2} s^{2}+a_{3}} \\
\frac{p_{0}^{L}(s)}{\gamma_{0}^{2} s^{2}+2 /\left(1-v_{0}\right)}
\end{array}\right\|,
$$

$$
\begin{aligned}
& w_{0, n}^{L}(s)=Q_{11}^{L}(n, s) p_{n}^{L}(s)+Q_{12}^{L}(n, s) q_{n}^{L}(s), \\
& u_{0, n}^{L}(s)=Q_{21}^{L}(n, s) p_{n}^{L}(s)+Q_{22}^{L}(n, s) q_{n}^{L}(s), \\
& \Phi_{n}^{L}(s)=Q_{31}^{L}(n, s) p_{n}^{L}(s)+Q_{32}^{L}(n, s) q_{n}^{L}(s),
\end{aligned}
$$

where

$Q_{i j}^{L}(n, s)=\frac{\left\|\begin{array}{c}\Delta_{i j}^{c y l} \\ \Delta_{i j}^{s p h}\end{array}\right\|(s)}{\Delta(s)},(i=\overline{1,3} ; j=1,2 ; n=\overline{1, \infty})$,

$\Delta_{21}^{c y l}(n, s)=-\beta_{4} n\left[a_{2}+\left(1+a_{4}\right)\left(\gamma_{0}^{2} s^{2}+n^{2}\right)\right]$,

$\Delta_{22}^{c y l}(n, s)=\beta_{3}\left[\left(\eta_{0}^{2} s^{2}+n^{2}\right)\left(\gamma_{0}^{2} s^{2}+n^{2}\right)+\right.$

$\left.+\left(\eta_{0}^{2} a_{2}+\gamma_{0}^{2} a_{3}\right) s^{2}+a_{3} n^{2}+a_{2} a_{3}\right]$,

$\Delta_{11}^{c y l}(n, s)=\beta_{4}\left[\left(\gamma_{0}^{2} s^{2}+n^{2}\right)\left(\gamma_{0}^{2} s^{2}+n^{2}\right)+a_{2}+a_{4}\right]$,

$\Delta_{12}^{c y l}(n, s)=-\beta_{3} n\left[\left(1+a_{3}\right)\left(\gamma_{0}^{2} s^{2}+n^{2}\right)+a_{2} a_{3}\right]$,

$\Delta_{31}^{c y l}(n, s)=\beta_{4} a_{2}\left[n\left(\gamma_{0}^{2} s^{2}+n^{2}\right)-1\right]$,

$\Delta_{32}^{c y l}(n, s)=\beta_{3} a_{2}\left[\eta_{0}^{2} s^{2}+a_{3}\left(1-n^{2}\right)\right]$,

$A_{a}=\left(\left(2 \eta_{0}^{2}+\gamma_{0}^{2}\right) n^{2}+a_{3} \gamma_{0}^{2}+a_{4} \eta_{0}^{2}\right) /\left(\eta_{0}^{2} \gamma_{0}^{2}\right)$,

$B_{b}=\left(\left(\eta_{0}^{2}+2 \gamma_{0}^{2}\right) n^{4}+\left(\left(a_{3}-2\right) \gamma_{0}^{2}+a_{4} \eta_{0}^{2}\right) n^{2}+\right.$

$\left.+a_{2}\left(\eta_{0}^{2} \gamma_{0}^{2}+a_{3} \gamma_{0}^{2}-a_{4} \eta_{0}^{2}\right)+\gamma_{0}^{2}\right) /\left(\eta_{0}^{2} \gamma_{0}^{4}\right)$,

$C_{c}=\left(n^{6}-2 n^{4}+\left(a_{2} \eta_{0}^{2}+1\right) n^{2}+a_{3} a_{4} \eta_{0}^{2}\right) /\left(\eta_{0}^{2} \gamma_{0}^{4}\right)$,

$\Delta_{11}^{s p h}(n, s)=\left(\frac{n(n+1)}{1-v_{0}^{2}}-\frac{1}{1-v_{0}}+\gamma_{0}^{2} s^{2}\right) \times$,

$\times\left(n(n+1)-1+v_{0}+R_{R}+\eta_{0}^{2} s^{2}\right)$,

$\Delta_{21}^{s p h}(n, s)=\left(R_{R}+\left(\frac{2\left(1+v_{0}\right) k_{s}}{1-v_{0}}+1\right)(n(n+1)-\right.$

$\left.\left.-1+v_{0}+R_{R}\right)+\eta_{0}^{2} s^{2}\right) /\left(2\left(1+v_{0}\right) D k_{s}\right)$,

$\Delta_{12}^{s p h}(n, s)=\frac{n(n+1)}{1-v_{0}}\left(n(n+1)-1+v_{0}+R_{R}+\eta_{0}^{2} s^{2}\right)$,

$\Delta_{22}^{s p h}(n, s)=-\frac{n(n+1) R_{R}}{2\left(1+v_{0}\right) k_{s}}+\left(\frac{n(n+1)}{2\left(1+v_{0}\right) k_{s}}+\right.$

$\left.+\frac{2}{1-v_{0}}+\gamma_{0}^{2} s^{2}\right)\left(n(n+1)-1+v_{0}+R_{R}+\eta_{0}^{2} s^{2}\right)$,

$\Delta_{31}^{s p h}(n, s)=\frac{R_{R}}{h}\left(-n(n+1)+\frac{1}{1+v_{0}}+\gamma_{0}^{2} s^{2}\right)$,

$\Delta_{32}^{s p h}(n, s)=-\frac{n(n+1) R_{R}}{\left(1-v_{0}\right) h}, R_{R}=\frac{R^{2} E_{0} h}{2\left(1+v_{0}\right) D k_{s}}$, 


$$
\begin{aligned}
& \Delta(s)=\eta_{0}^{2} \gamma_{0}^{4}\left(\left\|\begin{array}{c}
1 \\
-1 \|
\end{array}\right\| s^{6}+\left\|\begin{array}{c}
A_{a} \\
-\bar{A}_{a}
\end{array}\right\| s^{4}+\left\|\begin{array}{c}
B_{b} \\
-\bar{B}_{b}
\end{array}\right\| s^{2}+\left\|\begin{array}{c}
C_{c} \\
-\bar{C}_{c}
\end{array}\right\|\right), \quad \dot{w}_{0, n}(t)=\int_{0}^{t} p_{n}(\tau) Q_{11}(n, t-\tau) d \tau+ \\
& \bar{A}_{a}=\frac{1}{\gamma_{0}^{2}}\left(\frac{n(n+1)}{1-v_{0}^{2}}+\frac{n(n+1)}{2\left(1+v_{0}\right) k_{s}}+\frac{1}{1+v_{0}}+\right. \\
& \left.+\frac{2}{1-v_{0}}\right)+\frac{1}{\eta_{0}^{2}}\left(n(n+1)-1+v_{0}+\frac{R^{2} E_{0} h}{2\left(1+v_{0}\right) D k_{s}}\right) \\
& \bar{B}_{b}=\frac{1}{\eta_{0}^{2} \gamma_{0}^{4}}\left(\eta _ { 0 } ^ { 2 } ( \frac { n ( n + 1 ) } { 1 - v _ { 0 } ^ { 2 } } - \frac { 1 } { 1 + v _ { 0 } } ) \left(\frac{n(n+1)}{2\left(1+v_{0}\right) k_{s}}+\right.\right. \\
& \left.+\frac{2}{1-v_{0}}\right)+\gamma_{0}^{2}\left(\frac{n(n+1)}{1-v_{0}^{2}}+\frac{n(n+1)}{2\left(1+v_{0}\right) k_{s}}+\frac{1}{1+v_{0}}+\right. \\
& \left.+\frac{2}{1-v_{0}}\right)\left(n(n+1)-1+v_{0}+\frac{R^{2} E_{0} h}{2\left(1+v_{0}\right) k_{s}}+\frac{2}{1-v_{0}}\right)- \\
& -\frac{n(n+1)}{2\left(1+v_{0}\right) D k_{s}}\left(\frac{\eta_{0}^{2}}{\left(1-v_{0}\right)^{2}}\left(2\left(1+v_{0}\right) k_{s}+1-v_{0}\right)+\right. \\
& +\int_{0}^{t} q_{n}(\tau) Q_{12}(n, t-\tau) d \tau, \\
& \dot{u}_{0, n}(t)=\int_{0}^{t} p_{n}(\tau) Q_{21}(n, t-\tau) d \tau+ \\
& +\int_{0}^{t} q_{n}(\tau) Q_{22}(n, t-\tau) d \tau, \\
& \dot{\Phi}_{n}(t)=\int_{0}^{t} p_{n}(\tau) Q_{31}(n, t-\tau) d \tau+ \\
& +\int_{0}^{t} q_{n}(\tau) Q_{32}(n, t-\tau) d \tau, \quad(n=\overline{1, \infty})
\end{aligned}
$$$$
\left.\left.+\frac{\gamma_{0}^{2} R^{2} E_{0} h}{2\left(1+v_{0}\right) D k_{s}}\right)\right) \text {, }
$$$$
\bar{C}_{c}=\frac{1}{\eta_{0}^{2} \gamma_{0}^{4}}\left(( \frac { n ( n + 1 ) } { 1 - v _ { 0 } ^ { 2 } } - \frac { 1 } { 1 + v _ { 0 } } ) \left(\left(\frac{n(n+1)}{2\left(1+v_{0}\right) k_{s}}+\right.\right.\right.
$$$$
\left.+\frac{2}{1-v_{0}}\right)\left(n(n+1)-1+v_{0}+\frac{R^{2} E_{0} h}{2\left(1+v_{0}\right) D k_{s}}\right)-
$$$$
\left.-\frac{n(n+1) R^{2} E_{0} h}{4\left(1+v_{0}\right)^{2} D k_{s}^{2}}\right)+\frac{n(n+1)}{2\left(1-v_{0}^{2}\right) k_{s}} \times
$$$$
\times\left(\frac{R^{2} E_{0} h}{2\left(1+v_{0}\right) D k_{s}}-\left(\frac{2\left(1+v_{0}\right) k_{s}}{1-v_{0}}+1\right) \times\right.
$$$$
\left.\times\left(n(n+1)-1+v_{0}+\frac{R^{2} E_{0} h}{2\left(1+v_{0}\right) D k_{s}}\right)\right) \text {. }
$$

where

$$
\begin{aligned}
& Q_{i j}(n, t)=4\left[\left(\Delta_{r}\left\|\begin{array}{l}
R_{i j}^{c y l} \\
R_{i j}^{s p h} \|
\end{array}\right\|+\Delta_{i}\left\|\begin{array}{l}
I_{i j}^{c y l} \\
I_{i j}^{s p h}
\end{array}\right\|\right) \operatorname{ch}\left(r_{0} t\right) \cos \left(\sigma_{0} t\right)+\right. \\
& \left.+\left(\Delta_{i}\left\|\begin{array}{l}
R_{i j}^{c y l} \\
R_{i j}^{s p h}
\end{array}\right\|-\Delta_{r}\left\|\begin{array}{l}
I_{i j}^{c y l} \\
I_{i j}^{s p h} \|
\end{array}\right\|\right) \operatorname{sh}\left(r_{0} t\right) \sin \left(\sigma_{0} t\right)\right] /\left(\Delta_{r}^{2}+\Delta_{i}^{2}\right)+ \\
& +\frac{2 \Delta_{i j}\left(n, s_{1}^{2}\right)\left(\mathrm{H}\left(s_{1}^{2}\right) \operatorname{ch}\left(s_{1} t\right)+\mathrm{H}\left(-s_{1}^{2}\right) \cos \left(s_{1} t\right)\right)}{\Delta^{\prime}\left(s_{1}^{2}\right)},
\end{aligned}
$$

where $\mathrm{H}(\mathrm{x})$ - Heaviside function,

$r_{0}=\left(r^{2}+\sigma^{2}\right)^{1 / 4} \cos (\varphi / 2)$,

$\sigma_{0}=\left(r^{2}+\sigma^{2}\right)^{1 / 4} \sin (\varphi / 2), \varphi=\operatorname{arctg}(\sigma / r)$,

$r=-\left((A+B) / 2+\bar{A}_{a} / 3\right), \quad \sigma=\sqrt{3}(A-B) / 2$,

$s_{1}^{2}=A+B-\left\|A_{a}\right\| / 3, \quad A=\left(-q^{\prime} / 2+Q^{1 / 2}\right)^{1 / 3}$,

Then applying the inverse Laplace transform to $(28)-(41)$, by the theorem on the convolution of the originals of two functions, we have:

$$
\begin{aligned}
& \dot{w}_{0,0}(t)=\left\|\begin{array}{c}
\beta_{4} / \eta_{0}^{2} \\
1 / \gamma_{0}^{2}
\end{array}\right\| \int_{0}^{t} p_{0}(\tau) \times \\
& \times \cos \left(\left\|\begin{array}{c}
1 / \eta_{0}\left(a_{4}\right)^{1 / 2} \\
1 / \gamma_{0} \sqrt{\left(1-v_{0}\right) / 2}
\end{array}\right\|(t-\tau)\right) d \tau,
\end{aligned}
$$

$$
\begin{aligned}
& B=\left(-q^{\prime} / 2-Q^{1 / 2}\right)^{1 / 3}, Q=\left(p^{\prime} / 3\right)^{3}+\left(q^{\prime} / 2\right)^{2}, \\
& q^{\prime}=2\left(\left\|\begin{array}{l}
A_{a} \\
\bar{A}_{a}
\end{array}\right\| / 3\right)^{3}-\left\|\begin{array}{c}
A_{a} B_{b} \| \\
\bar{A}_{a} \bar{B}_{b}
\end{array}\right\| / 3+\left\|\begin{array}{l}
C_{c} \\
\bar{C}_{c}
\end{array}\right\|, \\
& p^{\prime}=-\left\|A_{a}^{2}\right\| / 3+\left\|\begin{array}{l}
B_{b} \\
\bar{A}_{a}^{2}
\end{array}\right\|, r_{1}=r^{2}-\sigma^{2}, \sigma_{1}=2 r \sigma, \\
& R_{b}^{c y l}=\beta_{4}\left[\gamma_{0}^{4} r_{1}+\gamma_{0}^{2}\left(2 n^{2}+a_{2}+a_{4}\right) r+\right. \\
& \left.+n^{2}\left(n^{2}+a_{2}+a_{4}\right)\right],
\end{aligned}
$$




$$
\begin{aligned}
& I_{11}^{c y l}=\beta_{4}\left[\gamma_{0}^{4} \sigma_{1}+\gamma_{0}^{2}\left(2 n^{2}+a_{2}+a_{4}\right) \sigma\right], \\
& R_{22}^{c y l}=\beta_{3}\left[\eta_{0}^{2} \gamma_{0}^{2} r_{1}+\left(\left(\gamma_{0}^{2}+\eta_{0}^{2}\right) n^{2}+\eta_{0}^{2} a_{2}+\gamma_{0}^{2} a_{3}\right) r+\right. \\
& \left.+n^{2}\left(n^{2}+a_{3}\right)+a_{2} a_{3}\right] \text {, } \\
& I_{22}^{c y l}=\beta_{3}\left[\eta_{0}^{2} \gamma_{0}^{2} \sigma_{1}+\left(\left(\gamma_{0}^{2}+\eta_{0}^{2}\right) n^{2}+\eta_{0}^{2} a_{2}+\gamma_{0}^{2} a_{3}\right) \sigma\right] \text {, } \\
& R_{12}^{c y l}=-\beta_{3} n\left[\left(1+a_{3}\right)\left(\gamma_{0}^{2} r+n^{2}\right)+a_{2} a_{3}\right], \\
& I_{12}^{c y l}=-\beta_{3} n\left(1+a_{3}\right) \gamma_{0}^{2} \sigma, \quad I_{21}=-\beta_{4} n\left(1+a_{4}\right) \gamma_{0}^{2} \sigma \text {, } \\
& R_{21}^{c y l}=-\beta_{4} n\left[\left(1+a_{4}\right)\left(\gamma_{0}^{2} r+n^{2}\right)+a_{2}\right] \text {, } \\
& R_{11}^{s p h}=\eta_{0}^{2} \gamma_{0}^{2} r_{1}+\left(\eta_{0}^{2}\left(\frac{n(n+1)}{1-v_{0}^{2}}-\frac{1}{1-v_{0}}\right)+\right. \\
& \left.+\gamma_{0}^{2}\left(n(n+1)-1+v_{0}+R_{R}\right)\right) r+ \\
& +\left(\frac{n(n+1)}{1-v_{0}^{2}}-\frac{1}{1-v_{0}}\right)\left(n(n+1)-1+v_{0}+R_{R}\right), \\
& I_{11}^{s p h}=\eta_{0}^{2} \gamma_{0}^{2} \sigma_{1}+\left(\eta_{0}^{2}\left(\frac{n(n+1)}{1-v_{0}^{2}}-\frac{1}{1-v_{0}}\right)+\right. \\
& \left.+\gamma_{0}^{2}\left(n(n+1)-1+v_{0}+R_{R}\right)\right) \sigma \text {, } \\
& R_{12}^{s p h}=\frac{n(n+1)}{1-v_{0}}\left(n(n+1)-1+v_{0}+R_{R}+\eta_{0}^{2} r\right), \\
& I_{12}^{s p h}=\eta_{0}^{2} \frac{n(n+1)}{1-v_{0}} \sigma, \\
& R_{21}^{s p h}=\frac{1}{2\left(1+v_{0}\right) D k_{s}}\left(\left(\frac{2\left(1+v_{0}\right) k_{s}}{1-v_{0}}+1\right) \times\right. \\
& \left.\times\left(n(n+1)-1+v_{0}+R_{R}+\eta_{0}^{2} r\right)-R_{R}\right), \\
& I_{21}^{s p h}=\eta_{0}^{2}\left(\frac{1}{1-v_{0}}+\frac{1}{2\left(1+v_{0}\right) k_{s}}\right) \sigma \text {, } \\
& R_{22}^{s p h}=\eta_{0}^{2} \gamma_{0}^{2} r_{1}+\left(\eta_{0}^{2}\left(\frac{n(n+1)}{2\left(1+v_{0}\right) k_{s}}+\frac{2}{1-v_{0}}\right)+\right. \\
& \left.+\gamma_{0}^{2}\left(n(n+1)-1+v_{0}+R_{R}\right)\right) r+ \\
& +\left(\frac{n(n+1)}{2\left(1+v_{0}\right) k_{s}}+\frac{2}{1-v_{0}}\right)\left(n(n+1)-1+v_{0}+R_{R}\right)- \\
& -\frac{n(n+1) R_{R}}{2\left(1+v_{0}\right) k_{s}}, \\
& I_{22}^{s p h}=\eta_{0}^{2} \gamma_{0}^{2} \sigma_{1}+\left(\eta_{0}^{2}\left(\frac{n(n+1)}{2\left(1+v_{0}\right) k_{s}}+\frac{2}{1-v_{0}}\right)+\right. \\
& \left.+\gamma_{0}^{2}\left(n(n+1)-1+v_{0}+R_{R}\right)\right) \sigma \text {, }
\end{aligned}
$$

$$
\begin{aligned}
& R_{31}^{s p h}=\frac{R_{R}}{h}\left(-n(n+1)+\frac{1}{1-v_{0}}+\gamma_{0}^{2} r\right), \\
& I_{31}^{s p h}=\gamma_{0}^{2} \frac{R_{R}}{h} \sigma, \quad R_{32}^{s p h}=-\frac{R_{R}}{h} n(n+1), \quad I_{32}^{s p h}=0, \\
& \Delta^{\prime}(s)=\eta_{0}^{2} \gamma_{0}^{4}\left[\left\|\begin{array}{c}
6 \\
-6
\end{array}\right\| s^{4}+4\left\|\begin{array}{c}
A_{a} \\
-\bar{A}_{a}
\end{array}\right\| s^{2}+2\left\|\begin{array}{c}
B_{b} \\
-\bar{B}_{b}
\end{array}\right\|\right], \\
& \Delta_{r}=\eta_{0}^{2} \gamma_{0}^{4}\left[\left\|\begin{array}{c}
6 \\
-6
\end{array}\right\| r_{1}+4\left\|\begin{array}{c}
A_{a} \\
-\bar{A}_{a}
\end{array}\right\| r+2\left\|\begin{array}{c}
B_{b} \\
-\bar{B}_{b}
\end{array}\right\|\right] \text {, } \\
& \Delta_{i}=\eta_{0}^{2} \gamma_{0}^{4}\left[\left\|\begin{array}{c}
6 \\
-6
\end{array}\right\| \sigma_{1}+4\left\|\begin{array}{c}
A_{a} \\
\bar{A}_{a}
\end{array}\right\| \sigma\right] \text {. } \\
& w_{0,0}(t)=\left\|\begin{array}{c}
\beta_{4}\left(a_{4}\right)^{1 / 2} / \eta_{0} \\
1 / \gamma_{0}
\end{array}\right\| \int_{0}^{t} p_{0}(\tau) \times \\
& \times \sin \left(\| \begin{array}{c}
1 / \eta_{0}\left(a_{4}\right)^{1 / 2} \\
1 / \gamma_{0} \sqrt{\left(1-v_{0}\right) / 2} \|(t-\tau)
\end{array}\right) d \tau \text {, } \\
& w_{0, n}(t)=\int_{0}^{t} p_{n}(\tau) \tilde{Q}_{11}(n, t-\tau) d \tau+ \\
& +\int_{0}^{t} q_{n}(\tau) \tilde{Q}_{12}(n, t-\tau) d \tau, \\
& u_{0, n}(t)=\int_{0}^{t} p_{n}(\tau) \tilde{Q}_{21}(n, t-\tau) d \tau+ \\
& +\int_{0}^{t} q_{n}(\tau) \tilde{Q}_{22}(n, t-\tau) d \tau, \\
& \Phi_{n}(t)=\int_{0}^{t} p_{n}(\tau) \tilde{Q}_{31}(n, t-\tau) d \tau+ \\
& +\int_{0}^{t} q_{n}(\tau) \tilde{Q}_{32}(n, t-\tau) d \tau, \quad(n=\overline{1, \infty})
\end{aligned}
$$

where

$\tilde{Q}_{i j}(n, t)=4\left[\left(\delta_{r}\left\|\begin{array}{l}R_{i j}^{c y l} \\ R_{i j}^{s p h}\end{array}\right\|+\delta_{i}\left\|\begin{array}{l}I_{i j}^{c y l} \\ I_{i j}^{s p h}\end{array}\right\|\right) \operatorname{sh}\left(r_{0} t\right) \cos \left(\sigma_{0} t\right)+\right.$ $\left.+\left(\delta_{i}\left\|\begin{array}{l}R_{i j}^{c y l} \\ R_{i j}^{s p h}\end{array}\right\|-\delta_{r}\left\|\begin{array}{l}I_{i j}^{c y l} \\ I_{i j}^{s p h}\end{array}\right\|\right) \operatorname{ch}\left(r_{0} t\right) \sin \left(\sigma_{0} t\right)\right] /\left(\delta_{r}^{2}+\delta_{i}^{2}\right)+$ $+\frac{2 \Delta_{i j}\left(n, s_{1}^{2}\right)\left(\mathrm{H}\left(s_{1}^{2}\right) \operatorname{sh}\left(s_{1} t\right)+\mathrm{H}\left(-s_{1}^{2}\right) \sin \left(s_{1} t\right)\right)}{\left(s_{1} \Delta^{\prime}\left(s_{1}^{2}\right)\right)}$,

$\delta_{r}=r_{0} \Delta_{r}-\sigma_{0} \Delta_{i}, \quad \delta_{i}=\sigma_{0} \Delta_{r}+r_{0} \Delta_{i}$.

We apply to the system of equations (2) and 
(3) the Laplace transform in the variable $t$ ( $s$ is the transformation parameter) and the Fourier method of separation of variables, considering the evenness in $x$ of the potential $\varphi$ and the oddness of the potential $\psi$, and require the satisfaction of condition (14) - (15). Then [2 $6]$, in the space of Laplace transformants, we obtain the following representations for wave potentials $[7-9,11]$ :

$$
\begin{aligned}
& \varphi^{L}\left(s, \| \begin{array}{l}
x \|, z)=\sum_{n=0}^{\infty} A_{n}(s) \times \\
r \|
\end{array}\right)\left\|\exp \left(-z \sqrt{\frac{s^{2}}{\alpha^{2}}+\lambda_{n}^{2}}\right)\right\| \begin{array}{c}
\cos \lambda_{n} x \\
J_{0}\left(\lambda_{n} r\right)
\end{array} \|, \\
& \psi^{L}\left(s, \| \begin{array}{l}
x \|, z \\
r \|
\end{array}\right)=\sum_{n=0}^{\infty} B_{n}(s) \times \\
& \times \exp \left(-z \sqrt{\frac{s^{2}}{\beta^{2}}+\lambda_{n}^{2}}\right)\left\|\begin{array}{c}
\sin \lambda_{n} x \\
J_{1}\left(\lambda_{n} r\right)
\end{array}\right\|,
\end{aligned}
$$

where $\lambda_{n}-$ the eigenvalues of the problem, which are determined from conditions (16) considering (5) in case of plane problem equal $\lambda_{n}=n \pi / l$, and in case of axisymmetric problem are the roots of the equality:

$$
J_{1}\left(\lambda_{n} l\right)=0, \quad(n=\overline{0, \infty}) .
$$

In (38) $A_{n}(s)$ and $B_{n}(s)$ are determined from the boundary conditions. It follows from representations (38) and relations (5) that the sought-for functions on the surface of a halfspace are represented as series in the system of eigenfunctions of the corresponding problems.

$$
\begin{aligned}
& \left.u_{z}\left(t,\left\|\begin{array}{l}
x \| \\
r
\end{array}\right\|, 0\right)=\sum_{n=0}^{\infty} u_{z n}(t) \| \begin{array}{l}
\cos \lambda_{n} x \\
J_{0}\left(\lambda_{n} r\right)
\end{array}\right), \\
& \left.u_{\|x\|}\left(t,\left\|\begin{array}{l}
x \| \\
r \|
\end{array}\right\|, 0\right)=\sum_{n=0}^{\infty} u_{\| x n}(t) \| \begin{array}{l}
\sin \lambda_{n} x \\
J_{1}\left(\lambda_{n} r\right)
\end{array}\right), \\
& \left.\sigma_{z z}\left(t,\left\|\begin{array}{l}
x \\
r \|
\end{array}\right\|, 0\right)=\sum_{n=0}^{\infty} \sigma_{z n}(t) \| \begin{array}{l}
\cos \lambda_{n} x \\
J_{0}\left(\lambda_{n} r\right)
\end{array}\right), \\
& \sigma_{\|z x\|}\left(t,\left\|\begin{array}{l}
x \| \\
z z r
\end{array}\right\|, 0\right)=\sum_{n=1}^{\infty} \sigma_{\left\|\begin{array}{l}
z x n \| \\
z r n
\end{array}\right\|}(t)\left\|\begin{array}{l}
\sin \lambda_{n} x \\
J_{1}\left(\lambda_{n} r\right)
\end{array}\right\| J_{1}\left(\lambda_{n} r\right) .
\end{aligned}
$$

Just as in [2-6], the dependence between the harmonics of the vertical component of the velocity and normal stresses on the surface of the half-space is determined $[7-9,11]$ :

$$
\sigma_{z n}(t)=-\alpha\left(V_{n}(t)+\int_{0}^{t} V_{n}(\tau) F(t-\tau) d \tau\right),
$$

where

$$
\begin{aligned}
& F_{n}(t)=-\alpha \lambda_{n} J_{1}\left(\alpha \lambda_{n} t\right)+2 b \beta \lambda_{n}\left\{\beta ^ { 2 } \lambda _ { n } ^ { 2 } t ^ { 2 } \left(\bar{J}_{0}\left(\alpha \lambda_{n} t\right)-\right.\right. \\
& \left.-\bar{J}_{0}\left(\beta \lambda_{n} t\right)-J_{1}\left(\alpha \lambda_{n} t\right)+J_{1}\left(\beta \lambda_{n} t\right)\right)+\beta \lambda_{n} t \times \\
& \times\left(b J_{0}\left(\alpha \lambda_{n} t\right)-J_{0}\left(\beta \lambda_{n} t\right)\right)+\left(2-b^{2}\right) \bar{J}_{0}\left(\alpha \lambda_{n} t\right)- \\
& \left.-\bar{J}_{0}\left(\beta \lambda_{n} t\right)\right\}
\end{aligned}
$$

where $J_{0}(t), J_{1}(t)$ - Bessel functions of the first kind of zero and first order, respectively, and the function $\bar{J}_{0}(t)$ is defined as follows:

$$
\bar{J}_{0}(t)=\int_{0}^{t} J_{0}(\tau) d \tau
$$

Further, we will satisfy the mixed boundary conditions (9). From (9), (39) we obtain the following representation for the vertical component of the velocity on the surface of the half-space:

$$
\begin{aligned}
& \sum_{n=0}^{\infty} V_{n}(t)\left\|\begin{array}{c}
\cos \lambda_{n} x \\
J_{0}\left(\lambda_{n} r\right)
\end{array}\right\|=\mathrm{H}\left(\left\|\begin{array}{c}
x^{*} \\
r^{*}
\end{array}\right\|-\left\|\begin{array}{c}
x \| \\
r
\end{array}\right\|\right) \times \\
& \left\{v_{T}(t)-\dot{w}_{0}(t, \theta) \cos \theta-\dot{u}_{0}(t, \theta) \sin \theta\right\}- \\
& -\mathrm{H}\left(\left\|\begin{array}{l}
x \| \\
r
\end{array}\right\|-\left\|\begin{array}{c}
x^{*} \\
r^{*}
\end{array}\right\|\right) \sum_{n=0}^{\infty}\left\|\begin{array}{c}
\cos \lambda_{n} x \\
J_{0}\left(\lambda_{n} r\right)
\end{array}\right\| \|_{0}^{t} V_{n}(\tau) F_{n}(t-\tau) d \tau .
\end{aligned}
$$

Substituting (23) and (24) into (40) with allowance for $\|x\|=\sin \theta$, arising from geometric considerations in the zone of the contact region, and representing both parts of (40) in the form of series in $\left\|\begin{array}{l}\cos \lambda_{n} x \\ J_{0}\left(\lambda_{n} r\right)\end{array}\right\|$, we obtain an infinite system of Volterra integral equations (ISVIE) of the second kind regarding to unknown harmonics velocity on the surface of the half-space $(n=\overline{0, \infty})$ : 


$$
\begin{aligned}
& V_{n}(t)+\sum_{m=0}^{\infty} \alpha_{m n}^{(\|1\|)}\left(\| \begin{array}{l}
x^{*} \\
r^{*}
\end{array}\right) \int_{0}^{t} V_{m}(\tau) F_{m}(t-\tau) d \tau+
\end{aligned}
$$

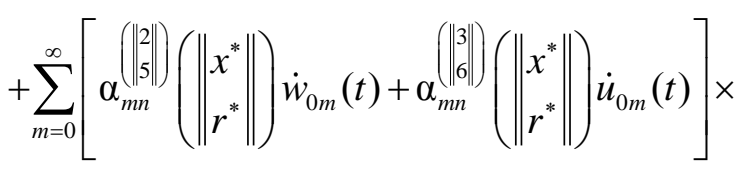

$$
\begin{aligned}
& \left.\times \int_{0}^{t} V_{m}(\tau) F_{m}(t-\tau) d \tau=C_{n}^{(\| 1} \begin{array}{l}
1 \\
2
\end{array}\right)\left(\left\|\begin{array}{l}
x^{*} \\
r^{*}
\end{array}\right\|\right) v_{T}(t),
\end{aligned}
$$

where

$\alpha_{m n}^{(1)}\left(x^{*}\right)=\frac{1}{N_{n}^{2}} \int_{x^{*}}^{l} \cos \lambda_{m} x \cos \lambda_{n} x d x$,

$\alpha_{m n}^{(2)}\left(x^{*}\right)=\frac{1}{N_{n}^{2}} \int_{0}^{x^{*}} \sqrt{1-x^{2}} D_{1 m}(x) \cos \lambda_{n} x d x$,

$\alpha_{m n}^{(3)}\left(x^{*}\right)=\frac{1}{N_{n}^{2}} \int_{0}^{x^{*}} x B_{1 m}(x) \cos \lambda_{n} x d x$

$C_{n}^{1}\left(x^{*}\right)=\frac{1}{N_{n}^{2}} \int_{0}^{x^{*}} \cos \lambda_{n} x d x, \quad N_{n}^{2}=\int_{0}^{l} \cos ^{2} \lambda_{n} x d x$,

$D_{1 m}(x)=\cos (m \pi / 2) T_{m}(x)+\sin (m \pi / 2) U_{m}(x)$,

$B_{1 m}(x)=\sin (m \pi / 2) T_{m}(x)-\cos (m \pi / 2) U_{m}(x)$,

$\alpha_{m n}^{(4)}\left(r^{*}\right)=\frac{1}{N_{n}^{2}} \int_{r^{*}}^{l} r J_{0}\left(\lambda_{m} r\right) J_{0}\left(\lambda_{n} r\right) d r$,

$\alpha_{m n}^{(5)}\left(r^{*}\right)=\frac{1}{N_{n}^{2}} \int_{0}^{r^{*}} r \sqrt{1-r^{2}} P_{m}\left(\sqrt{1-r^{2}}\right) J_{0}\left(\lambda_{n} r\right) d r$,

$\alpha_{m n}^{(6)}\left(r^{*}\right)=\frac{1}{K_{n}^{2}} \int_{0}^{r^{*}} r^{2} \sqrt{1-r^{2}} \frac{\partial}{\partial r} P_{m}\left(\sqrt{1-r^{2}}\right) J_{0}\left(\lambda_{n} r\right) d r$,

$C_{n}^{(2)}\left(r^{*}\right)=\frac{1}{K_{n}^{2}} \int_{0}^{r^{*}} r J_{0}\left(\lambda_{n} r\right) d r, K_{n}^{2}=\int_{0}^{l} r\left(J_{0}\left(\lambda_{n} r\right)\right)^{2} d r$.

here $T_{m}(x)$ and $U_{m}(x) \quad-$ Chebyshev polynomials of the first and second kind.

The functions $\dot{w}_{0 m}(t), \dot{u}_{0 m}(t)$ and $\dot{\Phi}_{n}(t)$ are determined from relations (32) - (35), but they involve unknown functions $p_{n}(t)$ and $q_{n}(t)$. Let us deal with their exclusion, for this we use conditions (7), (8), which can be rewritten using (39) in the form:

$$
\begin{aligned}
& \sum_{n=0}^{\infty} p_{n}(t)\left\|\begin{array}{c}
\cos n \theta \\
P_{n}(\cos \theta)
\end{array}\right\|=\alpha\left(\theta^{*}-|\theta|\right) \cos \theta \times \\
& \times \sum_{n=0}^{\infty}\left\|\begin{array}{c}
\cos \left(\lambda_{n} \sin \theta\right) \\
J_{0}\left(\lambda_{n} \sin \theta\right)
\end{array}\right\|\left(V_{n}(t)+\int_{0}^{t} V_{n}(\tau) F_{n}(t-\tau) d \tau\right), \\
& \sum_{n=0}^{\infty} q_{n}(t)\left\|\begin{array}{c}
\sin n \theta \\
P_{n}^{1}(\sin \theta) \|
\end{array}\right\|=\alpha \mathrm{H}\left(\theta^{*}-|\theta|\right) \sin \theta \times \\
& \times \sum_{n=0}^{\infty}\left\|\begin{array}{c}
\cos \left(\lambda_{n} \sin \theta\right) \\
J_{0}\left(\lambda_{n} \sin \theta\right)
\end{array}\right\|\left(V_{n}(t)+\int_{0}^{t} V_{n}(\tau) F_{n}(t-\tau) d \tau\right) .
\end{aligned}
$$

Using the orthogonality of the trigonometrical functions and the polynomials and the associated Legendre polynomials, we obtain the relations establishing the relationship between the harmonics of the series expansions of the functions $p, q$ and $V$ :

$$
\begin{aligned}
& p_{n}(t)=\sum_{m=0}^{\infty} \gamma_{m n}^{(\|1\|)}\left(\theta^{*}\right)\left(V_{m}(t)+\int_{0}^{t} V_{m}(\tau) F_{m}(t-\tau) d \tau\right), \\
& q_{n}(t)=\sum_{m=0}^{\infty} \gamma_{m n}^{(\|4\|)}\left(\theta^{*}\right)\left(V_{m}(t)+\int_{0}^{t} V_{m}(\tau) F_{m}(t-\tau) d \tau\right),
\end{aligned}
$$

where

$\gamma_{m n}^{(1)}\left(\theta^{*}\right)=\frac{\alpha}{\bar{N}_{n}^{2}} \int_{0}^{\theta^{*}} \cos \theta \cos n \theta \cos \left(\lambda_{m} \sin \theta\right) d \theta$, $\gamma_{m n}^{(2)}\left(\theta^{*}\right)=\frac{\alpha}{\tilde{N}_{n}^{2}} \int_{0}^{\theta^{*}} \sin \theta \sin n \theta \cos \left(\lambda_{m} \sin \theta\right) d \theta$, $\bar{N}_{n}^{2}=\int_{0}^{\pi} \cos ^{2} n \theta d \theta, \quad \tilde{N}_{n}^{2}=\int_{0}^{\pi} \sin ^{2} n \theta d \theta$.

$\gamma_{m n}^{(3)}\left(\theta^{*}\right)=\frac{\alpha}{\bar{K}_{n}^{2}} \int_{0}^{\theta^{*}} \cos \theta \sin \theta P_{n}(\cos \theta) J_{0}\left(\lambda_{m} \sin \theta\right) d \theta$,

$\gamma_{m n}^{(4)}\left(\theta^{*}\right)=\frac{\alpha}{\tilde{K}_{n}^{2}} \int_{0}^{\theta^{*}} \sin ^{2} \theta P_{n}^{1}(\cos \theta) J_{0}\left(\lambda_{m} \sin \theta\right) d \theta$,

$\gamma_{m n}^{(3)}\left(\theta^{*}\right)=\frac{\alpha}{\bar{K}_{n}^{2}} \int_{0}^{\theta^{*}} \cos \theta \sin \theta P_{n}(\cos \theta) J_{0}\left(\lambda_{m} \sin \theta\right) d \theta$,

$\gamma_{m n}^{(4)}\left(\theta^{*}\right)=\frac{\alpha}{\tilde{K}_{n}^{2}} \int_{0}^{\theta^{*}} \sin ^{2} \theta P_{n}^{1}(\cos \theta) J_{0}\left(\lambda_{m} \sin \theta\right) d \theta$,

$\bar{K}_{n}^{2}=\int_{0}^{\pi} \sin \theta\left(P_{n}(\cos \theta)\right)^{2} d \theta$ 


$$
\tilde{K}_{n}^{2}=\int_{0}^{\pi} \sin \theta\left(P_{n}^{1}(\cos \theta)\right)^{2} d \theta
$$

Thus, the final form of the resolving ISVIE of the second kind will be as follows:

$$
\begin{aligned}
& V_{n}(t)+\sum_{m=0}^{\infty}\left\|\begin{array}{l}
\alpha_{m n}^{(1)}\left(x^{*}\right) \\
\alpha_{m n}^{(4)}\left(r^{*}\right)
\end{array}\right\| \int_{0}^{t} V_{m}(\tau) F_{m}(t-\tau) d \tau+ \\
& \left.+\sum_{m=0}^{\infty}\left\|\begin{array}{l}
\alpha_{m n}^{(2)}\left(x^{*}\right) \\
\alpha_{m n}^{(5)}\left(r^{*}\right)
\end{array}\right\| \sum_{k=0}^{\infty} \int_{0}^{t} \gamma_{k m}^{(\| 1 / 1}\right)\left(\theta^{*}(\tau)\right)\left(V_{k}(\tau)+\right. \\
& \left.+\int_{0}^{\tau} V_{k}(\xi) F_{k}(\tau-\xi) d \xi\right) Q_{11}(m, t-\tau) d \tau+
\end{aligned}
$$

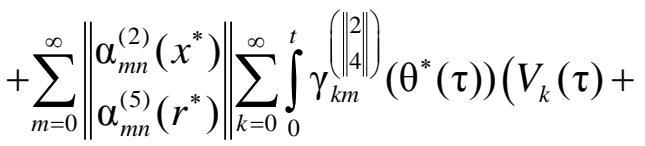

$$
\begin{aligned}
& \left.+\int_{0}^{\tau} V_{k}(\xi) F_{k}(\tau-\xi) d \xi\right) Q_{12}(m, t-\tau) d \tau+ \\
& \left.+\sum_{m=0}^{\infty} \| \begin{array}{l}
\alpha_{m n}^{(3)}\left(x^{*}\right) \\
\alpha_{m n}^{(6)}\left(r^{*}\right)
\end{array}\right) \| \sum_{k=0}^{\infty} \int_{0}^{t} \gamma_{k m}\left(\| \begin{array}{l}
1 \| \\
3
\end{array}\right)\left(\theta^{*}(\tau)\right)\left(V_{k}(\tau)+\right. \\
& \left.+\int_{0}^{\tau} V_{k}(\xi) F_{k}(\tau-\xi) d \xi\right) Q_{21}(m, t-\tau) d \tau+ \\
& +\sum_{m=0}^{\infty}\left\|\begin{array}{l}
\alpha_{m n}^{(3)}\left(x^{*}\right) \\
\alpha_{m n}^{(6)}\left(r^{*}\right)
\end{array}\right\| \sum_{k=0}^{\infty} \int_{0}^{t} \gamma_{k m}^{(\|2\|)}\left(\theta^{*}(\tau)\right)\left(V_{k}(\tau)+\right. \\
& \left.+\int_{0}^{\tau} V_{k}(\xi) F_{k}(\tau-\xi) d \xi\right) Q_{22}(m, t-\tau) d \tau= \\
& =C_{n}^{\| 1} \| \begin{array}{l}
12 \\
2
\end{array}\left(\| \begin{array}{l}
x^{*} \\
r^{*}
\end{array}\right) v_{T}(t), \quad(n=\overline{0, \infty}) .
\end{aligned}
$$

To solve the problem, when the shell penetration velocity $v_{T}(t)$ is a predetermined function, it is sufficient to numerically implement equations (41).

The expression for the reaction force of the elastic half-space (13), using (39), can be rewritten as:

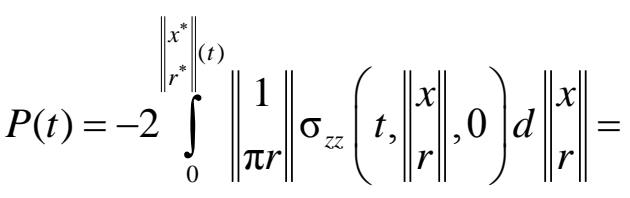

$$
\begin{aligned}
& =\alpha\left\|\begin{array}{c}
1 \\
\pi r^{*}(t)
\end{array}\right\|\left\{v_{T}(t)\left\|\begin{array}{c}
2 x^{*} \\
r^{*}
\end{array}\right\|(t)+\right. \\
& \left.+2 \sum_{n=0}^{\infty} \frac{1}{\lambda_{n}}\left\|\begin{array}{l}
\sin \lambda_{n} x^{*} \\
J_{1}\left(\lambda_{n} r^{*}\right)
\end{array}\right\| \int_{0}^{t} V_{n}(\tau) F_{n}(t-\tau) d \tau\right\} .
\end{aligned}
$$

The equation of motion of the shell (11) with the initial conditions takes the form:

$$
\begin{aligned}
& M \frac{d v_{T}(t)}{d t}=-\alpha\left(\left\|\begin{array}{c}
2 \\
\pi r^{*}(t)
\end{array}\right\|\right)\left\{v_{T}(t) r^{*}(t)+\right. \\
& \left.+\sum_{n=0}^{\infty} \frac{1}{\lambda_{n}}\left\|\begin{array}{c}
\sin \lambda_{n} x^{*} \\
2 J_{n}\left(\lambda_{n} r^{*}\right)
\end{array}\right\| \int_{0}^{t} V_{n}(\tau) F_{n}(t-\tau) d \tau\right\} .
\end{aligned}
$$

To solve the problem of impact with an initial velocity $V_{0}$, the system of equations (41) must be supplemented with the equation of motion (42).

The contact area is determined considering the rise of the medium from the condition:

$$
\begin{aligned}
& \delta_{1 j} v_{T} t+\delta_{2 j} \int_{0}^{t} v_{T}(\tau) d \tau-f\left(\left\|\begin{array}{l}
x^{*} \\
r^{*}
\end{array}\right\|\right)- \\
& -\sum_{n=0}^{\infty}\left\|\begin{array}{l}
\cos \lambda_{n} x^{*} \\
J_{0}\left(\lambda_{n} r^{*}\right)
\end{array}\right\| \int_{0}^{t} V_{n}(\tau) d \tau-
\end{aligned}
$$

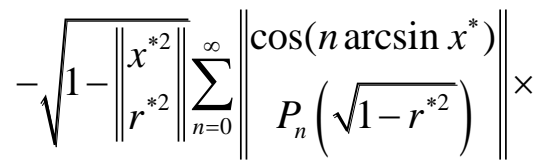

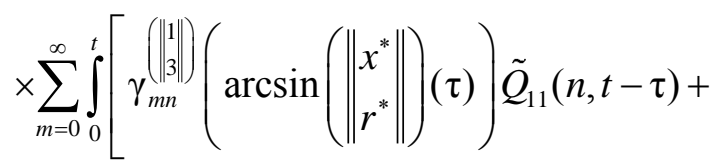

$$
\begin{aligned}
& \left.\left.+\gamma_{m n}^{(\|4\|} \|\right)\left(\arcsin \left(\left\|x^{*}\right\|\right)(\tau)\right) \tilde{Q}_{12}(n, t-\tau)\right] \times \\
& \times\left(V_{m}(\tau)+\int_{0}^{\tau} V_{m}(\xi) F_{m}(\tau-\xi) d \xi\right) d \tau-
\end{aligned}
$$




$$
\begin{aligned}
& -\left(\| \begin{array}{c}
x^{*} \\
r^{*} \sqrt{1-r^{* 2}}
\end{array}\right) \sum_{n=0}^{\infty}\left\|\begin{array}{c}
\sin \left(n \arcsin x^{*}\right) \\
P_{n}^{1}\left(\sqrt{1-r^{* 2}}\right)
\end{array}\right\| \times \\
& \times \sum_{m=0}^{\infty} \int_{0}^{t}\left[\left(\gamma_{m n}\left(\| \begin{array}{l}
1 \\
3
\end{array}\right)\left(\arcsin \left(\left\|\begin{array}{l}
x^{*} \\
r^{*}
\end{array}\right\|\right)(\tau)\right) \tilde{Q}_{21}(n, t-\tau)+\right.\right. \\
& \left.+\gamma_{m n}^{(\|4\|)} \arcsin \left(\left\|x^{*}\right\|\right)(\tau) \tilde{Q}_{22}(n, t-\tau)\right] \times \\
& \times\left(V_{m}(\tau)+\int_{0}^{\tau} V_{m}(\xi) F_{m}(\tau-\xi) d \xi\right) d \tau= \\
& =\left\{\begin{array}{c}
0, \quad \text { if } \quad\left(\left\|\begin{array}{c}
|x|<x^{*}(t) \| \\
r<r^{*}(t)
\end{array}\right\|\right) \\
\varepsilon<0, \quad \text { if }\left(\left\|\mid \begin{array}{c}
|x|>x^{*}(t) \\
r>r^{*}(t)
\end{array}\right\|\right)
\end{array} .\right.
\end{aligned}
$$

here $\delta_{i j}=\{0$, if $i \neq j ; 1$, if $i=j\}-$ Kronecker symbol. Index $j=1$ corresponds to the case when the body penetrates into the medium at a speed varying according to a predetermined law (setting 1); if the velocity of the penetrating body is known only at the initial moment of time $t=0$, and at subsequent moments is determined from the equation of motion (statement 2), then $j=2$. If we exclude the fourth term in relation (43), then we obtain a condition from which the boundary of the contact region is determined without considering the rise of the medium.

\section{NUMERICAL SOLUTION}

The size of reduction $N$ of the ISVIE of the second kind will be chosen from considerations of practical convergence. In case of plane problem

To smooth out the oscillations arising from the summation of a finite number of terms of the series, as well as Gibbs phenomena near points of weak discontinuity, the averaging operation was used, defined in [2-6], which, in the case of a sum of a finite number of terms of the trigonometric series, to memberwise multiplication of the members of the finite sum on $\sigma_{n}-$ Lanczos multipliers $[8,9,11]$. $\sigma_{n}=\left\{\begin{array}{lll}1, & \text { if } & n=0, \\ \frac{\sin (n \pi / N)}{n \pi / N}, & \text { if } & n \neq 0,\end{array}\right.$

The integrals were calculated using the method of mechanical quadratures, in particular, the symmetric Gregory quadrature formula for equidistant nodes. The Cauchy problem for the differential equation (52) was solved by the Adams method (closed-type formulas) [2-6] of order $m_{1}$ with a local truncation error $O\left(\Delta t^{m_{1}+1}\right) \quad[7-9,11]$. As a result of discretization, we obtain a system of linear algebraic equations (SLAE). Calculations have shown that with an increase in the reduction size $N$, the determinant of the SLAE matrix increases indefinitely. The SLAE is poorly defined: as the reduction size $N$ tends to infinity, the value of the determinant of the SLAE matrix also tends to infinity. This is due to the fact that the kernels $Q_{11}(n, t), Q_{22}(n, t)$ in (43), (44) have asymptotic $\exp (O(n))$ in the parameter $n, \tilde{Q}_{11}(n, t)$ and $\tilde{Q}_{22}(n, t)$ in (46) and (47) have asymptotic $O(1 / n) \exp (O(n))$ in the parameter $n$. Methods of Tikhonov regularization and orthogonal polynomials do not work to neutralize such an exponential singularity. The approach [1 - 5] for solving problems of dynamics makes it impossible to study the impact of elastic cylindric and spheric shells of the S.P. Timoshenko type and elastic bodies on an elastic foundation [7 - 9, 11]. In addition, this approach makes it possible to determine the stress-strain state only on the surface of the medium into which the striker penetrates.

\section{CONCLUSIONS}

As a result of an attempt to solve the plane and the axisymmetric problems of the impact of a cylindric and a spheric fine shells of the S.P. Timoshenko type on the surface of an elastic half-space, applying the method of reduction of dynamic problems to infinite systems of Voltaire's equations of the second kind, the limitations of this technique were 
revealed. This technique does not allow solving plane and axisymmetric [1] problems of dynamics for refined shells of the S.P. Timoshenko type and elastic bodies.

To solve [10, $12-15]$ the problems of impact and nonstationary interaction [16-20], the elastoplastic formulation [21 - 23] can be used. It should be noted that to calibrate the computational [2] process in the elastoplastic formulation at the elastic stage, it is convenient and expedient to use the technique [2-6] for solving the problems of dynamics, developed in $[7-9,11]$.

\section{REFERENCES}

1. Bogdanov V.R., 2021. One approach to the axisymmetric problem of impact of fine shells of the S.P. Timoshenko type on elastic halfspace. Transfer of Innovative Technologies. Vol.4 No.01, 68-74.

2. Bogdanov V.R., 2018. Impact a circular cylinder with a flat on an elastic layer. Transfer of Innovative Technologies, Vol.01(2), 68-74.

3. Bogdanov V.R., 2017. Impact of a hard cylinder with flat surface on the elastic layer. Underwater Technologies, Vol.05, 8-15.

4. Bogdanov V.R., Lewicki H.R., Pryhodko T.B., Radzivill O.Y., Samborska L.R., 2009. The planar problem of the impact shell against elastic layer. Visnyk NTU, Kyiv, No. 18, 281-292 (in Ukrainian).

5. Kubenko V.D., Bogdanov V.R., 1995. Planar problem of the impact of a shell on an elastic half-space. International Applied Mechanics, 31, No. 6, 483-490.

6. Kubenko V.D., Bogdanov V.R., 1995. Axisymmetric impact of a shell on an elastic halfspace. International Applied Mechanics, 31, No.10, 829-835.

7. Kubenko V.D., Popov S.N., Bogdanov V.R., 1995. The impact of elastic cylindrical shell with the surface of elastic half-space. Dop. NAN Ukrainy, No. 7, 40-44 (in Ukrainian).

8. Kubenko V.D., Popov S.N., 1988. Plane problem of the impact of hard blunt body on the surface of an elastic half-space. Pricl. Mechanika, 24, No.7, 69-77 (in Russian).

9. Popov S.N., 1989. Vertical impact of the hard circular cylinder lateral surface on the elastic half-space. Pricl. Mechanika, 25, No.12, 41-47 (in Russian).

10.Bogdanov V.R., Sulim G.T., 2016. Determination of the material fracture toughness by numerical analysis of 3D elastoplastic dynamic deformation. Mechanics of Solids, 51(2), 206215; DOI 10.3103/S0025654416020084.

11.Bogdanov V.R., 2015. A plane problem of impact of hard cylinder with elastic layer. Bulletin of University of Kyiv: Mathematics. Mechanics, No. 34, $42-47$ (in Ukrainian).

12.Bogdanov V.R., Sulym G.T., 2013. Plain deformation of elastoplastic material with profile shaped as a compact specimen (dynamic loading) // Mechanics of Solids, May, 48(4), 329-336; DOI 10.3103/S0025654413030096.

13.Bogdanov V.R., Sulym G.T., 2013. A modeling of plastic deformation's growth under impact, based on a numerical solution of the plane stress deformation problem // Vestnik Moskovskogo Aviatsionnogo Instituta, Volume 20, Iss. 3, 196-201 (in Russian).

14.Bogdanov V.R., 2009. Three dimension problem of plastic deformations and stresses concentration near the top of crack. // Bulletin of University of Kyiv: Series: Physics \& Mathematics, No. 2, 51-56 (in Ukrainian).

15.Bogdanov V.R., Sulym G.T., 2012. The plane strain state of the material with stationary crack with taking in account the process of unloading. Mathematical Methods and Physicomechanical Fields, Lviv, 55, No. 3, 132-138. (In Ukrainian)

16.Bogdanov V.R., Sulym G.T., 2010. The crack growing in compact specimen by plastic-elastic model of planar stress state. Bulletin of University of Kyiv: Series: Physics \& Mathematics, No.4, 58-62 (in Ukrainian).

17.Bogdanov V.R., Sulym G.T., 2010. The crack clevage simulation based on the numerical modelling of the plane stress state. Bulletin of University of Lviv: Series: Physics \& Mathematics, No.73, 192-204 (in Ukrainian).

18.Bohdanov V.R., Sulym G.T., 2011. Evaluation of crack resistance based on the numerical modelling of the plane strained state. Material Science, 46, No.6, 723-732.

19.Bogdanov V.R., Sulym G.T., 2011. The clevage crack simulation based on the numerical modelling of the plane deformation state. Scientific collection «Problems of Calculation Mechanics and Constructions Strength», Dnepropetrovsk, Nr. 15, 33-44 (in Ukrainian).

20.Bogdanov V.R., Sulym G.T., 2010. Destruction toughness determination based on the numerical modelling of the three dimension dynamic problem. International scientific collection «Strength of Machines and Constructions», Kyiv, No.43, 158-167 (in Ukrainian). 
21.Bogdanov V.R., Sulym G.T., 2012. A three dimension simulation of process of growing crack based on the numerical solution. Scientific collection «Problems of Calculation Mechanics and Constructions Strength», Dnepropetrovsk, No.19, 10-19 (in Ukrainian).

22.Bogdanov V.R., Sulym G.T., 2012. The crack clevage simulation in a compact specimen based on the numerical modelling of the three dimension problem. Scientific collection «Methods of Solving Applied Problems in Solid Mechanics», Dnepropetrovsk, No.13, 60-68 (in Ukrainian).

23.Bogdanov V.R., 2011. About three dimension deformation of an elastic-plastic material with the profile of compact shape. Theoretical and Applied Mechanics, Donetsk, No.3 (59), 51-58 (in Ukrainian).

24.Fedotenkov G.V., 2001. Cylinder shell impact along elastic semi-plane. Moscow Aviation Institute, 100 (in Russian).

25.Mihailova E.Y., $\quad$ Tarlakovsky D.V., Vahterova Y.A., 2018. generalized linear model of the dynamics of thin elastic shells. Scientific Notes of Kazan University, Series of Physics and Mathematics, 160(3), 561-577 (in Russian).

26.Lokteva N.A., Serduk D.O., Skopintsev P.D., Fedotenkov G.V., 2020. Non-stationary stressdeformed state of a composite cylindrical shell. Mechanics of Composite Materials and Structures, 26(4), 544-559; DOI: 10.33113/mkmk.ras.2020.26.04.544_559.08 (in Russian).

27.Vestyak A.V., Igumnov L.A., Tarlakovskii D.V., Fedotenkov G.V., 2016. The influence of non-stationary pressure on a thin spherical shell with an elastic filler. Computational Continuum Mechanics. 9(4), 443-452. DOI: 10.7242/19996691/2016.9.4.37 (in Russian).

28.Afanasyeva O.A. Mikhailova E.Y. Fedotenkov G.V., 2012. Random phase of contact interaction of a spherical shell and elastic half space. Problems of Computer Mechanics and Strength of Structures, 20, 19-26 (in Russian).

29.Michailova E.Y., Tarlakovsky D.V., Fedotenkov G.V., 2014. Non-stationary contact of a spherical shell and an elastic half-space. Works MAI. 78, 1-26 (in Russian).

30.Igumnov L.A., Okonechnikov A.S., Tarlakovskii D.V., Fedotenkov G.V. 2013. Plane nonsteady-state problem of motion of the surface load on an elastic half-space. Mathematical Methods and Physicomechanical Fields, Lviv, 56, Nr. 2, 157-163 (in Russian).
31.Kuznetsova E.L., Tarlakovsky D.V., Fedotenkov G.V., Medvedsky A.L., 2013. Influence of non-stationary distributed load on the surface of the elastic layer. Works MAI. 71, 1-21 (in Russian).

32.Fedotenkov G.V., Tarlakovsky D.V., Vahterova Y.A., 2019. Identification of Non-stationary Load Upon Timoshenko Beam. Lobachevskii Journal of Mathematics, 40(4), 439-447.

33.Vahterova Y.A., Fedotenkov G.V., 2020. The inverse problem of recovering an unsteady linear load for an elastic rod of finite length. Journal of Applied Engineering Science, 18(4), 687-692, DOI:10.5937/jaes0-28073.

34.Gorshkov A.G., $\quad$ Tarlakovsky D.V., 1985. Dynamic contact problems with moving boundaries. Nauka, Fizmatlit, 352 (in Russian).

35.Sagomonian A.J., 1985. Stress waves in a continuous medium. Moscow University Publishing House, 416 (in Russian).

\section{Один подход к задачам удара оболочек типа С.П. Тимошенко об упругое полупространство}

\section{Владислав Богданов}

Аннотация. Оболочечные элементы
используются во многих тонкостенных
конструкция. Поэтому изучение динамики
распространения волновых процессов в тонких
оболочках типа С.П. Тимошенко является
важным аспектом, так же как важно
исследование волновых процессов удара в
упругом основании, в которое проникает
ударник. Уточненная модель С.П. Тимошенко,
описывающая динамику оболочек, позволяет
учесть сдвиг и инерцию вращения поперечного
сечения оболочки. Хорошо изучены метод
сведения решения задач динамики к решению
бесконечной системы интегральных уравнений
Вольтерра второго рода и сходимость этого
решения. Такой подход успешно применялся
для случаев исследования задач об ударе
твердых тел и упругих тонких оболочек типа
Кирхгофа - Лява об упругие полупространство
и слой. В данной работе решается плоская и
осесиммет-ричная задачи об ударе упругих
тонких цилиндрической и сферической
оболочек типа С.П. Тимошенко об упругое
полупространство методом сведения задач
динамики к решению бесконечной системы
интегральных уравнений Вольтерра второго
рода. В результате попытки решения таким


методом показано, что такой подход неприемлем для исследуемых в данной статье плоской и осесимметричной задач. Дискретизация с использованием методов Грегори для численного интегрирования и Адамса для решения задачи Коши для полученной бесконечной системы уравнений Вольтерра второго рода приводит к решению плохо определенной системы линейных алгебраических уравнений: при увеличении порядка редукции определитель такой системы стремиться к бесконечности. Показано, что данная методика не позволяет решать плоские и осесимметричные задачи динамики для тонких оболочек типа С.П. Тимошенко и упругих тел.
Это является ограниченностью такого подхода и показывает необходимость разработки других математических подходов и моделей. Следует отметить, что для калибровки вычислительного процесса в динамической упругопластической постановке на упругом этапе процесса деформирования удобно и целесообразно использовать технику сведения задач динамики к решению бесконечной системы интегральных уравнений Вольтерра второго рода.

Ключевые слова: удар, упругость, упругопластичность, полупространство, осесимметричная задача, тонкая сферическая оболочка, С.П. Тимошенко. 